\title{
THE SECOND REGULARIZED TRACE FORMULA FOR THE STURM-LIOUVILLE OPERATOR
}

\author{
F. AYDIN AKGUN, M. BAYRAMOGLU, AND A. BAYRAMOV
}

Received 02 May, 2018

\begin{abstract}
In this paper, the second regularized trace formula for the differential operator with antiperiodic boundary conditions is obtained.
\end{abstract}

2010 Mathematics Subject Classification: 34L20; 35R10

Keywords: eigenvalue, eigenfunction, resolvent, regularized trace

\section{INTRODUCTION}

In the Hilbert space $H=L_{2}[0, \pi]$, we consider operator $\mathrm{L}$ generated by the differential expression

$$
l(y)=-y^{\prime \prime}+q(x) y,
$$

with the antiperiodic boundary conditions

$$
y(0)=-y(\pi), y^{\prime}(0)=-y^{\prime}(\pi),
$$

where $q(x) \in C^{2}[0, \pi]$ is real function and satisfies the condition

$$
q(0)=q(\pi)
$$

It is well known from [13] that the eigenvalues of the operator $L$ form double series

$$
\lambda_{n, j}=\left(2 n-1+\frac{c_{0}}{2 n-1}+o\left(\frac{1}{n}\right)\right)^{2},
$$

where $c_{0}=\frac{1}{2 \pi} \int_{0}^{\pi} q(x) d x, j=1,2, o\left(\frac{1}{n}\right)$ include $j$ and $n=1,2, \ldots$. Let $L_{0}$ denote the operator $L$ with $q(x) \equiv 0$. The eigenvalues of operator $L_{0}$ are

$$
\mu_{n}=(2 n-1)^{2}, \quad n=1,2, \ldots .
$$

The orthonormal eigenfunctions corresponding to the eigenvalues $\mu_{n}$ are

$$
\psi_{n}^{1}=\sqrt{\frac{2}{\pi}} \cos (2 n-1) x, \psi_{n}^{2}=\sqrt{\frac{2}{\pi}} \sin (2 n-1) x, n=1,2, \ldots
$$


It is natural to define second regularized trace of operator $L$ as

$$
\sum_{n=1}^{\infty} \prime\left(\lambda_{n, 1}^{2}+\lambda_{n, 2}^{2}-2(2 n-1)^{4}\right)
$$

where the symbol "," means that something in this sum is discarded to provide its convergence. The sum in (1.3) is the main interest of this article.

The regularized trace formula

$$
\sum_{n=0}^{\infty}\left(\lambda_{n}-n^{2}-\frac{1}{\pi} \int_{0}^{\pi} q(x) d x\right)=\frac{q(0)+q(\pi)}{4}-\frac{1}{2 \pi} \int_{0}^{\pi} q(x) d x,
$$

of the Stum-Liouvile operator

$$
-y^{\prime \prime}+q(x) y=\lambda y, y^{\prime}(0)=0, y^{\prime}(\pi)=0,
$$

with $q(x) \in C^{1}[0, \pi]$ was first studied by Gelfand and Levitan ([6]), where the $\lambda_{n}$ are the eigenvalues of the operator in (1.4). Afterwards, trace formulas for different differential operators are studied by several mathematicians(see [1-5,8,10-12,14,15] and references therein).

Note that the first regularized trace formula

$$
\sum_{n=0}^{\infty}\left[\lambda_{n, 1}+\lambda_{n, 2}-2(2 n-1)^{2}-2 c_{0}\right]=0
$$

was obtained for operator $L$ with a real potential $q(x) \in L_{2}(0, \pi)$, by [10] and with an arbitrary complex $q(x) \in L_{2}(0, \pi)$, by [15].The similar formula was obtained by [12] for the operator $L$ with operator function $q(x)$.

Trace formulas are used in inverse problems of spectral analysis of differential equations(see [14]) and for approximate calculation of the first eigenvalues of the related operator $[1,4,5,7,9,14]$.

\section{SOME FORMULAS ABOUT THE OPERATOR $q R_{\lambda}^{0}$}

Let $R_{\lambda}^{0}$ and $R_{\lambda}$ be the resolvents of the operator $L_{0}$ and $L$, respectively. Then, for any $\lambda \in \rho(L)$ and $\mu \in \rho\left(L_{0}\right)$ where $\rho\left(\right.$.) is the resolvent set of an operator, $R_{\lambda}: H \rightarrow$ $H$ and $R_{\lambda}^{0}: H \rightarrow H$ are trace class operators, that is, $R_{\lambda} R_{\lambda}^{0} \in \sigma_{1}(H)$. Therefore,

$$
\operatorname{tr}\left(R_{\lambda}-R_{\lambda}^{0}\right)=\sum_{n=1}^{\infty}\left(\frac{1}{\lambda_{n, 1}-\lambda}+\frac{1}{\lambda_{n, 2}-\lambda}-\frac{2}{(2 n-1)^{2}-\lambda}\right) .
$$

Multiplying both sides of the above equality by $\lambda^{2} / 2 \pi i$ then integrating over the circle $|\lambda|=b_{p}=(2 p-1)^{2}+4 p$,

$$
\frac{1}{2 \pi i} \int_{|\lambda|=b_{p}} \lambda^{2} \operatorname{tr}\left(R_{\lambda}-R_{\lambda}^{0}\right) d \lambda=\sum_{n=1}^{p}\left(2(2 n-1)^{4}-\lambda_{n, 1}^{2}-\lambda_{n, 2}^{2}\right)
$$


is obtained. Taking in the account that $R_{\lambda}-R_{\lambda}^{0}=-R_{\lambda} q R_{\lambda}^{0}$, from equation (2.1),

$$
\sum_{n=1}^{p}\left(\lambda_{n, 1}^{2}+\lambda_{n, 2}^{2}-2(2 n-1)^{4}\right)=\sum_{j=1}^{N} M_{p}^{j}+M_{p N}
$$

is obtained. Here

$$
M_{p}^{j}=\frac{(-1)^{j+1}}{2 \pi i} \int_{|\lambda|=b_{p}} \lambda^{2} \operatorname{tr}\left[R_{\lambda}^{0}\left(q R_{\lambda}^{0}\right)^{j}\right] d \lambda,
$$

and

$$
M_{p N}=\frac{(-1)^{N}}{2 \pi i} \int_{|\lambda|=b_{p}} \lambda^{2} \operatorname{tr}\left[R_{\lambda}\left(q R_{\lambda}^{0}\right)^{N+1}\right] d \lambda,
$$

where $q=q(x)$ and $N$ is an integer.

The formula

$$
M_{p}^{j}=\frac{(-1)^{j}}{2 \pi i j} \int_{|\lambda|=b_{p}} \lambda \operatorname{tr}\left(q R_{\lambda}^{0}\right)^{j} d \lambda,
$$

can be proved similarly as in Theorem 2 in [12].

From equations (1.2) and (2.4), we write

$$
\begin{aligned}
M_{p}^{1} & =-\frac{1}{\pi i} \int_{|\lambda|=b_{p}}\left\{\lambda \sum_{n=1}^{\infty}\left[\left(q R_{\lambda}^{0} \psi_{n}^{1}, \psi_{n}^{1}\right)+\left(q R_{\lambda}^{0} \psi_{n}^{2}, \psi_{n}^{2}\right)\right]\right\} d \lambda \\
& =2 \sum_{n=1}^{\infty}\left[\left(q \psi_{n}^{1}, \psi_{n}^{1}\right)+\left(q \psi_{n}^{2}, \psi_{n}^{2}\right)\right] \frac{1}{2 \pi i} \int_{|\lambda|=b_{p}} \frac{\lambda}{\lambda-\mu_{n}} d \lambda \\
& =\frac{4}{\pi} \sum_{n=1}^{p}(2 n-1)^{2} \int_{0}^{\pi} q(x)\left[\cos ^{2}(2 n-1) x+\sin ^{2}(2 n-1) x\right] d x \\
& =\frac{4}{\pi} \sum_{n=1}^{p}(2 n-1)^{2} \int_{0}^{\pi} q(x) d x .
\end{aligned}
$$

Now, we shall compute $M_{p}^{2}$. From equation (2.4),

$$
\begin{aligned}
M_{p}^{2} & =\frac{1}{2 \pi i} \int_{|\lambda|=b_{p}} \lambda\left\{\sum_{n=1}^{\infty}\left[\left(\left(q R_{\lambda}^{0}\right)^{2} \psi_{n}^{1}, \psi_{n}^{1}\right)+\left(\left(q R_{\lambda}^{0}\right)^{2} \psi_{n}^{2}, \psi_{n}^{2}\right)\right]\right\} d \lambda \\
& =\frac{1}{2 \pi i} \int_{|\lambda|=b_{p}} \lambda\left\{\sum_{n=1}^{\infty} \frac{1}{\mu_{n}-\lambda}\left[\left(q R_{\lambda}^{0} q \psi_{n}^{1}, \psi_{n}^{1}\right)+\left(q R_{\lambda}^{0} q \psi_{n}^{2}, \psi_{n}^{2}\right)\right]\right\} d \lambda \\
& =\frac{1}{2 \pi i} \int_{|\lambda|=b_{p}} \lambda\left\{\sum _ { n = 1 } ^ { \infty } \sum _ { r = 1 } ^ { \infty } \frac { 1 } { ( \mu _ { n } - \lambda ) ( \mu _ { r } - \lambda ) } \left[\left(q \psi_{n}^{1}, \psi_{r}^{1}\right)\left(q \psi_{r}^{1}, \psi_{n}^{1}\right)\right.\right. \\
& \left.\left.+\left(q \psi_{n}^{1}, \psi_{r}^{2}\right)\left(q \psi_{r}^{2}, \psi_{n}^{1}\right)+\left(q \psi_{n}^{2}, \psi_{r}^{1}\right)\left(q \psi_{r}^{1}, \psi_{n}^{2}\right)+\left(q \psi_{n}^{2}, \psi_{r}^{2}\right)\left(q \psi_{r}^{2}, \psi_{n}^{2}\right)\right]\right\} d \lambda .
\end{aligned}
$$


For convenience, let

$$
q_{n r}=\left|\left(q \psi_{n}^{1}, \psi_{r}^{1}\right)\right|^{2}+\left|\left(q \psi_{n}^{1}, \psi_{r}^{2}\right)\right|^{2}+\left|\left(q \psi_{n}^{2}, \psi_{r}^{1}\right)\right|^{2}+\left|\left(q \psi_{n}^{2}, \psi_{r}^{2}\right)\right|^{2} .
$$

Then,

$$
\begin{aligned}
M_{p}^{2}= & \sum_{n=1}^{\infty} \sum_{r=1}^{\infty} q_{n r} \frac{1}{2 \pi i} \int_{|\lambda|=b_{p}} \frac{\lambda}{\left(\lambda-\mu_{n}\right)\left(\lambda-\mu_{r}\right)} d \lambda \\
= & \sum_{n=1}^{p} \sum_{r=1}^{p} q_{n r} \frac{1}{2 \pi i} \int_{|\lambda|=b_{p}} \frac{\lambda}{\left(\lambda-\mu_{n}\right)\left(\lambda-\mu_{r}\right)} d \lambda \\
& +\sum_{n=1}^{p} \sum_{r=p+1}^{\infty} q_{n r} \frac{1}{2 \pi i} \int_{|\lambda|=b_{p}} \frac{\lambda}{\left(\lambda-\mu_{n}\right)\left(\lambda-\mu_{r}\right)} d \lambda \\
& +\sum_{n=p+1}^{\infty} \sum_{r=1}^{p} q_{n r} \frac{1}{2 \pi i} \int_{|\lambda|=b_{p}} \frac{\lambda}{\left(\lambda-\mu_{n}\right)\left(\lambda-\mu_{r}\right)} d \lambda \\
& +\sum_{n=p+1}^{\infty} \sum_{r=p+1}^{\infty} q_{n r} \frac{1}{2 \pi i} \int_{|\lambda|=b_{p}} \frac{\left.\lambda-\mu_{n}\right)\left(\lambda-\mu_{r}\right)}{(\lambda-\lambda} \\
= & \sum_{n=1}^{p} \sum_{r=1}^{p} q_{n r}+2 \sum_{n=1}^{p} \sum_{r=p+1}^{\infty} q_{n r} \frac{\mu_{n}}{\mu_{r}-\mu_{n}} \\
= & \sum_{n=1}^{p} \sum_{r=1}^{\infty} q_{n r}-\sum_{n=1}^{p} \sum_{r=p+1}^{\infty} \frac{\mu_{r}+\mu_{n}}{\mu_{r}-\mu_{n}} q_{n r} .
\end{aligned}
$$

Thus,

$$
M_{p}^{2}=\sum_{n=1}^{p} \sum_{r=1}^{\infty} q_{n r}-\alpha_{p}
$$

where

$$
\alpha_{p}=\sum_{n=1}^{p} \sum_{r=p+1}^{\infty} \frac{\mu_{r}+\mu_{n}}{\mu_{r}-\mu_{n}} q_{n r}
$$

By using equations (1.2) and (2.6), we have

$$
\alpha_{p}=\alpha_{p 1}+\alpha_{p 2}+\alpha_{p 3},
$$

where

$$
\alpha_{p 1}=2 \pi^{-2} \sum_{n=1}^{p} \sum_{r=1}^{\infty} \frac{(2 r-1)^{2}+(2 n-1)^{2}}{(2 r-1)^{2}-(2 n-1)^{2}}\left\{\left|\int_{0}^{\pi} q(x) \cos 2(n-r) x d x\right|^{2}+\right.
$$




$$
\begin{gathered}
\left.+\left|\int_{0}^{\pi} q(x) \sin 2(n-r) x d x\right|^{2}\right\}, \\
\alpha_{p 2}=2 \pi^{-2} \sum_{n=1}^{p} \sum_{r=1}^{\infty} \frac{(2 r-1)^{2}+(2 n-1)^{2}}{(2 r-1)^{2}-(2 n-1)^{2}}\left|\int_{0}^{\pi} q(x) \cos 2(n+r-1) x d x\right|^{2}, \\
\alpha_{p 3}=2 \pi^{-2} \sum_{n=1}^{p} \sum_{r=1}^{\infty} \frac{(2 r-1)^{2}+(2 n-1)^{2}}{(2 r-1)^{2}-(2 n-1)^{2}}\left|\int_{0}^{\pi} q(x) \sin 2(n+r-1) x d x\right|^{2} .
\end{gathered}
$$

The formula of $\alpha_{p 1}$ can be written as

$$
\begin{gathered}
\alpha_{p 1}=2 \pi^{-2} \sum_{i=1}^{\infty} \sum_{\substack{r-n=i \\
n \leq p \\
r>p}}\left(1+\frac{2(2 n-1)^{2}}{(2 r-1)^{2}-(2 n-1)^{2}}\right) \\
\left\{\left|\int_{0}^{\pi} q(x) \cos 2 i x d x\right|^{2}+\left|\int_{0}^{\pi} q(x) \sin 2 i x d x\right|^{2}\right\} .
\end{gathered}
$$

For $i \leq p$

$$
\begin{aligned}
\sum_{\substack{c r-n=i \\
n \leq p \\
r>p}} & \frac{(2 n-1)^{2}}{(2 r-1)^{2}-(2 n-1)^{2}}=\sum_{j=0}^{i-1} \frac{(2(p-j)-1)^{2}}{(2(p-j+i)-1)^{2}-(2(p-j)-1)^{2}} \\
& =\sum_{j=0}^{i-1} \frac{(2(p-j)-1+i)^{2}-2(2(p-j)-1+i) i+i^{2}}{4 i(2(p-j)-1+i)} \\
& =\frac{2 p-1}{4}+\frac{1-2 i}{4}+\sum_{j=0}^{i-1} \frac{i}{4(2 p-1-2 j+i)} .
\end{aligned}
$$

For any integers $p$ and $i$, let

$$
E=\{(r, n): r, n \in N ; r-n=i ; n \leq p ; r>p\} .
$$

Then using (2.11), we write

$$
\begin{gathered}
\sum_{n, r \in E}\left(1+\frac{2(2 n-1)^{2}}{(2 r-1)^{2}-(2 n-1)^{2}}\right)=i+2 \sum_{\substack{c r-n=i \\
n \leq p \\
r>p}} \frac{(2 n-1)^{2}}{(2 r-1)^{2}-(2 n-1)^{2}} \\
=p+\frac{i}{2} \sum_{j=p-i+1}^{p} \frac{1}{2 j-1+i}, \quad(i \leq p) .
\end{gathered}
$$


It is easy to see that

$$
\frac{i}{2} \sum_{j=p-i+1}^{p} \frac{1}{2 j-1+i}<\frac{i^{2}}{p} .
$$

Using this inequality and equation (2.12),

$$
\sum_{n, r \in E}\left(1+\frac{2(2 n-1)^{2}}{(2 r-1)^{2}-(2 n-1)^{2}}\right)=p+i^{2} O\left(p^{-1}\right), \quad(i \leq p)
$$

is obtained.

Here $O\left(p^{-1}\right)$ depends on $p$ and $i$, and satisfies the inequality

$$
\left|O\left(p^{-1}\right)\right|<\text { const. } p^{-1} \text {. }
$$

In a similar form, for $i \geq p$, it can be shown that

$$
\sum_{n, r \in E}\left(1+\frac{2(2 n-1)^{2}}{(2 r-1)^{2}-(2 n-1)^{2}}\right)=O(p) .
$$

Here $O(p)$ depends on $p$ and $i$, and satisfies the inequality

$$
|O(p)|<\text { const.p. }
$$

From (2.9), (2.13) and (2.14), we obtain

$$
\begin{aligned}
\alpha_{p 1}= & 2 \pi^{-2} p \sum_{i=1}^{\infty}\left\{\left|\int_{0}^{\pi} q(x) \cos 2 i x d x\right|^{2}+\left|\int_{0}^{\pi} q(x) \sin 2 i x d x\right|^{2}\right\} \\
& +\sum_{i=1}^{p} i^{2} O\left(p^{-1}\right)\left\{\left|\int_{0}^{\pi} q(x) \cos 2 i x d x\right|^{2}+\left|\int_{0}^{\pi} q(x) \sin 2 i x d x\right|^{2}\right\} \\
& +\sum_{i=p+1}^{\infty} O(p)\left\{\left|\int_{0}^{\pi} q(x) \cos 2 i x d x\right|^{2}+\left|\int_{0}^{\pi} q(x) \sin 2 i x d x\right|^{2}\right\} \\
= & \frac{p}{\pi} \int_{0}^{\pi}|q(x)|^{2} d x-\frac{p}{\pi^{2}}\left|\int_{0}^{\pi} q(x) d x\right|^{2}+\alpha_{p 1}^{(1)}+\alpha_{p 1}^{(2)} .
\end{aligned}
$$

Here, since $q(0)=q(\pi)$,

$$
\begin{aligned}
\left|\alpha_{p 1}^{(1)}\right|= & \left|\sum_{i=1}^{p} i^{2} O\left(p^{-1}\right)\left\{\left|\int_{0}^{\pi} q(x) \cos 2 i x d x\right|^{2}+\left|\int_{0}^{\pi} q(x) \sin 2 i x d x\right|^{2}\right\}\right| \\
& \leq \text { const. } p^{-1} \int_{0}^{\pi}\left|q^{\prime}(x)\right|^{2} d x
\end{aligned}
$$


and

$$
\begin{aligned}
\left|\alpha_{p 1}^{(2)}\right|= & \left|\sum_{i=p+1}^{\infty} O(p)\left\{\left|\int_{0}^{\pi} q(x) \cos 2 i x d x\right|^{2}+\left|\int_{0}^{\pi} q(x) \sin 2 i x d x\right|^{2}\right\}\right| \\
& \leq \text { const. } p^{-1} \int_{0}^{\pi}\left|q^{\prime}(x)\right|^{2} d x
\end{aligned}
$$

is obtained. From (2.15)-(2.17), we get

$$
\alpha_{p 1}=\frac{p}{\pi} \int_{0}^{\pi} q^{2}(x) d x-\frac{p}{\pi^{2}}\left|\int_{0}^{\pi} q(x) d x\right|^{2}+O\left(p^{-1}\right) .
$$

Since $q(x)$ satisfies the condition in equation (1.1), it can be shown that

$$
\left|\alpha_{p j}\right| \leq \text { const. }\left(p^{-1}\right), \quad(j=2,3) .
$$

From equation (2.6), we have

$$
\sum_{n=1}^{p} \sum_{r=1}^{\infty} q_{n r}=\sum_{n=1}^{p}\left\{\left\|q \psi_{n}^{1}\right\|^{2}+\left\|q \psi_{n}^{2}\right\|^{2}\right\}=\frac{2 p}{\pi} \int_{0}^{\pi} q^{2}(x) d x .
$$

From equations (2.7), (2.8) and (2.18)-(2.20),

$$
M_{p}^{2}=\frac{p}{\pi} \int_{0}^{\pi} q(x)^{2} d x+\frac{p}{\pi^{2}}\left(\int_{0}^{\pi} q(x) d x\right)^{2}+O\left(p^{-1}\right),
$$

is obtained.

\section{THE SECOND REGULARIZED TRACE FORMULA}

In this section we obtain the second regularized trace formula for the operator $L$. To do this, we will first show that the formulas

$$
\begin{array}{ll}
\lim _{p \rightarrow \infty} M_{p}^{j}=0, & j \geq 3, \\
\lim _{p \rightarrow \infty} M_{p}^{N}=0, & N \geq 6,
\end{array}
$$

are satisfied.

The inequalities

$$
\left\|q R_{\lambda}^{0}\right\|_{\sigma_{1}(H)}<C,\left\|R_{\lambda}^{0}\right\|<C p^{-1},\left\|R_{\lambda}\right\|<C p^{-1}, \quad\left(|\lambda|=b_{p}\right)
$$

are true(see [12]). Here $C>0$ is a constant. From (2.4) and (3.3), we have

$$
\begin{aligned}
\left|M_{p}^{j}\right|= & \frac{1}{\pi j}\left|\int_{|\lambda|=b_{p}} \lambda \operatorname{tr}\left(q R_{\lambda}^{0}\right)^{j} d \lambda\right| \\
& \leq \frac{b_{p}}{\pi j} \int_{|\lambda|=b_{p}}\left\|\left(q R_{\lambda}^{0}\right)^{j}\right\|_{\sigma_{1}(H)}|d \lambda|
\end{aligned}
$$




$$
\begin{aligned}
& \leq \frac{b_{p}}{\pi j} \int_{|\lambda|=b_{p}}\left\|\left(q R_{\lambda}^{0}\right)\right\|_{\sigma_{1}(H)}\left\|\left(q R_{\lambda}^{0}\right)^{j-1}\right\||d \lambda| \\
& \leq \frac{C b_{p}}{\pi j} \int_{|\lambda|=b_{p}}\|q\|^{j-1}\left\|\left(R_{\lambda}^{0}\right)\right\|^{j-1}|d \lambda| \\
& <C_{1} p^{5-j}\left(C_{1}>0\right) .
\end{aligned}
$$

This implies that

$$
\lim _{p \rightarrow \infty} M_{p}^{j}=0, \quad(j \geq 6)
$$

but we claim that it is also true for $j=3,4,5$. Now let we prove the formula (3.1) for $j=3$.

$$
\begin{aligned}
M_{p}^{3}= & -\frac{1}{3 \pi i} \sum_{n=1}^{\infty} \sum_{r=1}^{\infty} \sum_{k=1}^{\infty} \int_{|\lambda|=b_{p}} \frac{\lambda d \lambda}{\left(\mu_{n}-\lambda\right)\left(\mu_{r}-\lambda\right)\left(\mu_{k}-\lambda\right)} \\
& *\left\{\left(\left(q \psi_{n}^{1}, \psi_{r}^{1}\right)\left[\left(q \psi_{r}^{1}, \psi_{k}^{1}\right) \psi_{k}^{1}+\left(q \psi_{r}^{1}, \psi_{k}^{2}\right) \psi_{k}^{2}\right]\right.\right. \\
& \left.+\left(q \psi_{n}^{1}, \psi_{r}^{2}\right)\left[\left(q \psi_{r}^{2}, \psi_{k}^{1}\right) \psi_{k}^{1}+\left(q \psi_{r}^{2}, \psi_{k}^{2}\right) \psi_{k}^{2}\right], \psi_{n}^{1}\right) \\
& +\left(\left(q \psi_{n}^{2}, \psi_{r}^{1}\right)\left[\left(q \psi_{r}^{1}, \psi_{k}^{1}\right) \psi_{k}^{1}+\left(q \psi_{r}^{1}, \psi_{k}^{2}\right) \psi_{k}^{2}\right]\right. \\
& \left.\left.+\left(q \psi_{n}^{2}, \psi_{r}^{2}\right)\left[\left(q \psi_{r}^{2}, \psi_{k}^{1}\right) \psi_{k}^{1}+\left(q \psi_{r}^{2}, \psi_{k}^{2}\right) \psi_{k}^{2}\right], \psi_{n}^{2}\right)\right\} \\
= & -\frac{1}{3 \pi i} \sum_{n=1}^{\infty} \sum_{r=1}^{\infty} \sum_{k=1}^{\infty} \int_{|\lambda|=b_{p}} \frac{\lambda d \lambda}{\left(\mu_{n}-\lambda\right)\left(\mu_{r}-\lambda\right)\left(\mu_{k}-\lambda\right)} F(n, r, k),
\end{aligned}
$$

where

$$
\begin{aligned}
F(n, r, k)= & \left(q \psi_{n}^{1}, \psi_{r}^{1}\right)\left(q \psi_{r}^{1}, \psi_{k}^{1}\right)\left(q \psi_{k}^{1}, \psi_{n}^{1}\right)+\left(q \psi_{n}^{1}, \psi_{r}^{1}\right)\left(q \psi_{r}^{1}, \psi_{k}^{2}\right)\left(q \psi_{k}^{2}, \psi_{n}^{1}\right) \\
& +\left(q \psi_{n}^{1}, \psi_{r}^{2}\right)\left(q \psi_{r}^{2}, \psi_{k}^{1}\right)\left(q \psi_{k}^{1}, \psi_{n}^{1}\right)+\left(q \psi_{n}^{1}, \psi_{r}^{2}\right)\left(q \psi_{r}^{2}, \psi_{k}^{2}\right)\left(q \psi_{k}^{2}, \psi_{n}^{1}\right) \\
& +\left(q \psi_{n}^{2}, \psi_{r}^{1}\right)\left(q \psi_{r}^{1}, \psi_{k}^{1}\right)\left(q \psi_{k}^{1}, \psi_{n}^{2}\right)+\left(q \psi_{n}^{2}, \psi_{r}^{1}\right)\left(q \psi_{r}^{1}, \psi_{k}^{2}\right)\left(q \psi_{k}^{2}, \psi_{n}^{2}\right) \\
& +\left(q \psi_{n}^{2}, \psi_{r}^{2}\right)\left(q \psi_{r}^{2}, \psi_{k}^{1}\right)\left(q \psi_{k}^{1}, \psi_{n}^{2}\right)+\left(q \psi_{n}^{2}, \psi_{r}^{2}\right)\left(q \psi_{r}^{2}, \psi_{k}^{2}\right)\left(\psi_{k}^{2}, \psi_{n}^{2}\right) .
\end{aligned}
$$


Since $F(n, r, k)=F(r, n, k)=F(k, n, r)=F(k, r, n)=F(n, k, r)=F(r, k, n)$, from equation (3.4)

$$
\begin{aligned}
M_{p}^{3}= & \frac{1}{\pi i} \sum_{n=1}^{p} \sum_{r=p+1}^{\infty} \sum_{k=p+1}^{\infty} \int_{|\lambda|=b_{p}} \frac{\lambda d \lambda}{\left(\lambda-\mu_{n}\right)\left(\lambda-\mu_{r}\right)\left(\lambda-\mu_{k}\right)} F(n, r, k) \\
& +\frac{1}{\pi i} \sum_{n=1}^{p} \sum_{\substack{r=1 \\
n \neq r}}^{p} \sum_{k=p+1}^{\infty} \int_{|\lambda|=b_{p}} \frac{\lambda d \lambda}{\left(\lambda-\mu_{n}\right)\left(\lambda-\mu_{r}\right)\left(\lambda-\mu_{k}\right)} F(n, r, k) \\
& +\frac{1}{\pi i} \sum_{n=1}^{p} \sum_{k=p+1}^{\infty} \int_{|\lambda|=b_{p}} \frac{\lambda d \lambda}{\left(\lambda-\mu_{n}\right)\left(\lambda-\mu_{k}\right)} F(n, n, k) \\
= & 2 \sum_{n=1}^{p} \sum_{r=p+1}^{\infty} \sum_{k=p+1}^{\infty} \frac{\mu_{n}}{\left(\mu_{r}-\mu_{n}\right)\left(\mu_{k}-\mu_{n}\right)} F(n, r, k) \\
& +4 \sum_{n=1}^{p} \sum_{c r=1}^{p} \sum_{\substack{n \neq r \\
n=p+1}}^{\infty} \frac{\mu_{n}}{\left(\mu_{n}-\mu_{r}\right)\left(\mu_{n}-\mu_{k}\right)} F(n, r, k) \\
& -2 \sum_{n=1}^{p} \sum_{k=p+1}^{\infty} \frac{\mu_{k}}{\left(\mu_{k}-\mu_{n}\right)^{2}} F(n, n, k)
\end{aligned}
$$

is obtained. Let

$$
\begin{aligned}
F_{1}(n, r, k)= & \pi^{-3} \int_{0}^{\pi} q(x) \cos 2(n-r) x d x \\
& \int_{0}^{\pi} q(x) \cos 2(r-k) x d x \int_{0}^{\pi} q(x) \cos 2(k-n) x d x, \\
A_{p i}= & \sum_{n=1}^{p} \sum_{r=p+1}^{\infty} \sum_{k=p+1}^{\infty} \frac{\mu_{n}}{\left(\mu_{r}-\mu_{n}\right)\left(\mu_{k}-\mu_{n}\right)} F_{i}(n, r, k), \\
B_{p i}= & \sum_{n=1}^{p} \sum_{r=1}^{p} \sum_{k=p+1}^{\infty} \frac{\mu_{n}}{\left(\mu_{n}-\mu_{r}\right)\left(\mu_{n}-\mu_{k}\right)} F_{i}(n, r, k), \\
C_{p i}= & \sum_{n=1}^{p} \sum_{k=p+1}^{\infty} \frac{\mu_{k}}{\left(\mu_{k}-\mu_{n}\right)^{2}} F_{i}(n, n, k), \quad(i=1,2) .
\end{aligned}
$$

From equation (3.6), we obtain

$$
F_{1}(n, r, k)=F_{1}(n, k, r), \quad F_{1}(n, n, k)=F_{1}(n, k, k) .
$$


Using these equalities and equation (3.8), we have

$$
\begin{aligned}
A_{p 1}= & \sum_{n=1}^{p} \sum_{c r=p+1}^{\infty} \sum_{\substack{r>k \\
r=p+1}}^{\infty} \frac{\mu_{n}}{\left(\mu_{r}-\mu_{n}\right)\left(\mu_{k}-\mu_{n}\right)} F_{1}(n, r, k) \\
& +\sum_{n=1}^{p} \sum_{k=p+1}^{\infty} \frac{\mu_{n}}{\left(\mu_{k}-\mu_{n}\right)^{2}} F_{1}(n, n, k) .
\end{aligned}
$$

In similar way, it can be shown that,

$$
B_{p 1}=-\sum_{n=1}^{p} \sum_{\substack{c r=1 \\ n<r}}^{p} \sum_{k=p+1}^{\infty} \frac{\mu_{k}}{\left(\mu_{r}-\mu_{n}\right)\left(\mu_{k}-\mu_{n}\right)} F_{1}(n, r, k) .
$$

Let

$$
\begin{aligned}
& A_{p}^{1}=\sum_{n=1}^{p} \sum_{\substack{c=p+1 \\
r>k}}^{\infty} \sum_{k=p+1}^{\infty} \frac{\mu_{n}}{\left(\mu_{r}-\mu_{n}\right)\left(\mu_{k}-\mu_{n}\right)} F_{1}(n, r, k), \\
& B_{p}^{1}=-B_{p 1}, \\
& C_{p}^{1}=\sum_{n=1}^{p} \sum_{k=p+1}^{\infty} \frac{1}{\mu_{k}-\mu_{n}} F_{1}(n, n, k) .
\end{aligned}
$$

Hence, by using equations (3.5)-(3.15), we obtain

$$
M_{p}^{3}=4 A_{p}^{1}-4 B_{p}^{1}-2 C_{p}^{1}+2 A_{p 2}+4 B_{p 2}-2 C_{p 2} .
$$

Let us find a formula for $A_{p}^{1}$.

Let

$$
E_{1}=\{(n, r, k): n, r, k \in N ; r-n=i ; k-n=j ; n \leq p ; r, k>p\},
$$

where $p, i$ and $j$ are integers such that $p \geq j, i \geq j$, then

$$
\begin{aligned}
A_{p}^{1}= & \sum_{n=1}^{p} \sum_{\substack{r=p+1 \\
r>k}}^{\infty} \sum_{\substack{k=p+1 \\
k-n \leq p}}^{\infty} \frac{\mu_{n}}{\left(\mu_{r}-\mu_{n}\right)\left(\mu_{k}-\mu_{n}\right)} F_{1}(n, r, k) \\
& +\sum_{n=1}^{p} \sum_{\substack{r=p+1 \\
r>k}}^{\infty} \sum_{\substack{k=p+1 \\
k-n>p}}^{\infty} \frac{\mu_{n}}{\left(\mu_{r}-\mu_{n}\right)\left(\mu_{k}-\mu_{n}\right)} F_{1}(n, r, k) \\
= & \pi^{-3} \sum_{\substack{i=2 \\
i>j}}^{\infty} \sum_{j=1}^{p}\left[\sum_{n, r, k \in E_{1}} \frac{\mu_{n}}{\left(\mu_{r}-\mu_{n}\right)\left(\mu_{k}-\mu_{n}\right)} \int_{0}^{\pi} q(x) \cos 2 i x d x\right.
\end{aligned}
$$




$$
\begin{aligned}
& \left.\times \int_{0}^{\pi} q(x) \cos 2(i-j) x d x \int_{0}^{\pi} q(x) \cos 2 j x d x\right] \\
& +\sum_{n=1}^{p} \sum_{\substack{r=p+1 \\
r>k}}^{\infty} \sum_{\substack{k=p+1 \\
k-n>p}}^{\infty} \frac{\mu_{n}}{\left(\mu_{r}-\mu_{n}\right)\left(\mu_{k}-\mu_{n}\right)} F_{1}(n, r, k) .
\end{aligned}
$$

Let

$$
\beta_{i j}=\pi^{-3} \int_{0}^{\pi} q(x) \cos 2 i x d x \int_{0}^{\pi} q(x) \cos 2(i-j) x d x \int_{0}^{\pi} q(x) \cos 2 j x d x,
$$

and

$$
\begin{aligned}
& A_{p}^{11}=\sum_{i=2}^{\infty} \sum_{\substack{i>1 \\
i>j}}^{p}\left[\left(\sum_{n, r, k \in E_{1}} \frac{\mu_{n}}{\left(\mu_{r}-\mu_{n}\right)\left(\mu_{k}-\mu_{n}\right)}\right) \beta_{i j}\right], \\
& A_{p}^{12}=\sum_{n=1}^{p} \sum_{\substack{r=p+1 \\
r>k}}^{\infty} \sum_{\substack{k=p+1 \\
k-n>p}}^{\infty} \frac{\mu_{n}}{\left(\mu_{r}-\mu_{n}\right)\left(\mu_{k}-\mu_{n}\right)} F_{1}(n, r, k),
\end{aligned}
$$

then we write

$$
A_{p}^{1}=A_{p}^{11}+A_{p}^{12} .
$$

By similar proof of (2.14), it can be shown that

$$
\begin{aligned}
& \sum_{n, r, k \in E_{1}} \frac{\mu_{n}}{\left(\mu_{r}-\mu_{n}\right)\left(\mu_{k}-\mu_{n}\right)}= \\
= & \sum_{n, r, k \in E_{1}} \frac{(2 n-1)^{2}}{\left((2 r-1)^{2}-(2 n-1)^{2}\right)\left((2 k-1)^{2}-(2 n-1)^{2}\right)} \\
= & \frac{1}{16 i}+\frac{j}{p} O(1),
\end{aligned}
$$

where $O(1)$ satisfies the condition

$$
|O(1)|<\text { const. }
$$

and depends on $p, i$ and $j$. Moreover, if $q(x)$ has a continuous derivative of second order at $[0, \pi]$ and satisfies the condition in (1.1), then it can be shown that

$$
\beta_{i j}=\frac{\text { const }}{i^{2} j^{2}} .
$$

From (3.18), (3.20) and (3.21),

$$
A_{p}^{11}=\sum_{\substack{i=2 \\ i>j}}^{\infty} \sum_{j=1}^{p}\left[\frac{\beta_{i j}}{16 i}+\frac{O(1)}{p i^{2} j}\right],
$$


is obtained.

Since

$$
\left|\sum_{i=2}^{\infty} \sum_{j=1}^{p} \frac{O(1)}{p i^{2} j}\right| \leq \text { const. } p^{-1}\left(\sum_{i=1}^{\infty} i^{-2}\right) \sum_{j=1}^{p} j^{-1}<\text { const. } p^{-1} \ln p,
$$

we find

$$
A_{p}^{11}=\sum_{\substack{i=2 \\ i>j}}^{\infty} \sum_{\substack{j=1 \\ \text { p }}}^{p} \frac{\beta_{i j}}{16 i}+o(1) .
$$

Here $o(1)$ is an expression which satisfies the condition

$$
\lim _{p \rightarrow \infty} o(1)=0,
$$

and depends on $p$. From 3.19 and 3.22, we obtain

$$
A_{p}^{1}=\sum_{\substack{i=2 \\ i>j}}^{\infty} \sum_{\substack{j=1 \\ 16 i}}^{p} \beta_{i j} A_{p}^{12}+o(1)
$$

Now, to find the formula for $B_{p}^{1}$, let

$$
B_{p}^{11}=\sum_{\substack{n=1 \\ n<r, k-n \leq p}}^{p} \sum_{\substack{r=1 \\ n=p+1}}^{\infty} \frac{\mu_{k}}{\left(\mu_{k}-\mu_{n}\right)\left(\mu_{k}-\mu_{r}\right)} F_{1}(n, r, k)
$$

and

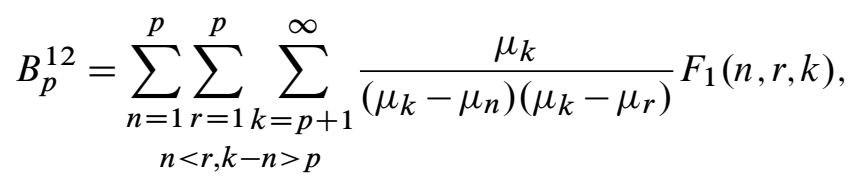

then from (3.12) and (3.14), we have

$$
B_{p}^{1}=B_{p}^{11}+B_{p}^{12} .
$$

By using equations in (3.6) and (3.7), $B_{p}^{11}$ can be written as

$$
\left.B_{p}^{11}=\sum_{\substack{j=2 \\ j>i}}^{p} \sum_{\substack{i=1 \\ j>i}}^{p-1} \sum_{n, r, k \in E_{2}} \frac{\mu_{k}}{\left(\mu_{k}-\mu_{n}\right)\left(\mu_{k}-\mu_{r}\right)}\right) \beta_{i j},
$$

where $E_{2}$ is a set defined by

$$
E_{2}=\{(n, r, k): n, r, k \in N ; r-n=i ; k-n=j ; n, r \leq p ; k>p\}
$$


for $i<j \leq p$. Moreover it can be shown that

$$
\sum_{n, r, k \in E_{2}} \frac{\mu_{k}}{\left(\mu_{k}-\mu_{n}\right)\left(\mu_{k}-\mu_{r}\right)}=\frac{1}{16 j}+\frac{i}{p} O(1) .
$$

From (3.21), (3.25) and (3.26), we obtain

$$
B_{p}^{11}=\sum_{\substack{j=2 \\ j>i}}^{p} \sum_{\substack{i=1 \\ j-1}}^{p-1}\left[\frac{\beta_{i j}}{16 j}+\frac{O(1)}{{p j^{2} i}^{2}}\right] .
$$

and, since $\beta_{i j}=\beta j i$, we write

$$
B_{p}^{11}=\sum_{\substack{j=2 \\ j>i}}^{p} \sum_{\substack{i=1 \\ j>i}}^{p-1} \frac{\beta_{i j}}{16 j}+O(1) .
$$

By using above equation and 3.24, we have

$$
B_{p}^{1}=\sum_{\substack{i=2 \\ i>j}}^{p} \sum_{\substack{j=1 \\ \text { p }}}^{p-1} \frac{\beta_{i j}}{16 i}+B_{p}^{12}+o(1) .
$$

From (3.24), we get

$$
\begin{gathered}
\left|\sum_{i=2}^{p} \frac{\beta_{i p}}{i}\right| \leq \sum_{i=2}^{p} \frac{1}{i^{3} p^{2}}=o(1), \\
\left|\sum_{i=p+1}^{\infty} \sum_{j=2}^{p} \frac{\beta_{i j}}{i}\right| \leq \sum_{i=p+1}^{\infty} \sum_{j=2}^{p} \frac{1}{i^{3} j^{2}}=\left(\sum_{i=p+1}^{\infty} \frac{1}{i^{3}}\right)\left(\sum_{j=1}^{p} \frac{1}{j^{2}}\right)=o(1) .
\end{gathered}
$$

Therefore, by (3.27), we have

$$
B_{p}^{1}=\sum_{\substack{i=2 \\ i>j}}^{\infty} \sum_{\substack{j=1 \\ 16 i}}^{p}+B_{p}^{12}+o(1) .
$$

From (3.16), (3.23) and (3.28), we obtain

$$
M_{p}^{3}=4 A_{p}^{12}-4 B_{p}^{12}-2 C_{p}^{1}+2 A_{p 2}+4 B_{p 2}-2 C_{p 2}+o(1) .
$$

Here, it can be easily seen that,

$$
\lim _{p \rightarrow \infty} A_{p}^{12}=\lim _{p \rightarrow \infty} B_{p}^{12}=\lim _{p \rightarrow \infty} C_{p}^{1}=\lim _{p \rightarrow \infty} A_{p 2}=\lim _{p \rightarrow \infty} B_{p 2}=\lim _{p \rightarrow \infty} C_{p 2}=0 .
$$


From (3.29) and (3.30), we obtain

$$
\lim _{p \rightarrow \infty} M_{p}^{3}=0 .
$$

In a similar way, it can be proved that

$$
\lim _{p \rightarrow \infty} M_{p}^{4}=\lim _{p \rightarrow \infty} M_{p}^{5}=0 .
$$

Now, let us prove the expression given in (3.2). By employing (2.2) and (3.3), we obtain

$$
\begin{aligned}
\left|M_{p N}\right|= & \frac{1}{2 \pi}\left|\int_{|\lambda|=b_{p}} \lambda^{2} \operatorname{tr}\left[R_{\lambda}\left(q R_{\lambda}^{0}\right)^{N+1}\right] d \lambda\right| \\
& \leq \frac{b_{p}^{2}}{2 \pi} \int_{|\lambda|=b_{p}}\left\|R_{\lambda}\left(q R_{\lambda}^{0}\right)^{N+1}\right\|_{\sigma_{1}(H)}|d \lambda| \\
& \leq b_{p}^{2} \int_{|\lambda|=b_{p}}\left\|R_{\lambda}\right\|\left\|\left(q R_{\lambda}^{0}\right)^{N+1}\right\|_{\sigma_{1}(H)}|d \lambda| \\
& \leq C b_{p}^{2} p^{-1} \int\left\|q R_{\lambda}^{0}\right\|^{N}\left\|\left(q R_{\lambda}^{0}\right)\right\|_{\sigma_{1}(H)}|d \lambda| \\
& \leq \text { const. } p^{5-N} .
\end{aligned}
$$

This shows that

$$
\lim _{p \rightarrow \infty} M_{p N}=0, \quad N \geq 6 .
$$

By using the equations (2.2), (2.5), (3.1) and (3.2), we find

$$
\begin{aligned}
\sum_{n=1}^{p}\left(\lambda_{n, 1}^{2}+\lambda_{n, 2}^{2}-2(2 n-1)^{4}\right)= & \frac{4}{\pi} \sum_{n=1}^{p}(2 n-1)^{2} \int_{0}^{\pi} q(x) d x \\
& +\frac{p}{\pi} \int_{0}^{\pi} q^{2}(x) d x+\frac{p}{\pi^{2}}\left(\int_{0}^{\pi} q(x) d x\right)^{2}+o(1) .
\end{aligned}
$$

As a result, we get

$$
\begin{gathered}
\sum_{n=1}^{p}\left[\lambda_{n, 1}^{2}+\lambda_{n, 2}^{2}-2(2 n-1)^{4}-\frac{4}{\pi}(2 n-1)^{2} \int_{0}^{\pi} q(x) d x\right. \\
\left.-\frac{1}{\pi} \int_{0}^{\pi} q^{2}(x) d x-\frac{1}{\pi^{2}}\left(\int_{0}^{\pi} q(x) d x\right)^{2}\right]=0 .
\end{gathered}
$$


The left hand-side of this equality is called the second regularized trace of the operator $L$. Thus we have proven the main result of this article given by the following theorem.

Theorem 1. If $q(x) \in C^{2}[0, \pi]$ is a real function which satisfies the condition (1.1), then the equality obtained in (3.31) holds for the second regularized trace of the operator $L$.

\section{REFERENCES}

[1] N. M. Aslanova, " $n$-th regularized trace of differential operator equation." Trans. Natl. Acad. Sci. Azerb., Ser. Phys.-Tech. Math. Sci., vol. 26, no. 7, Math. Mech., pp. 27-32, 2006.

[2] M. Bayramoglu, A. Bayramov, and E. Şen, "A regularized trace formula for a discontinuous Sturm-Liouville operator with delayed argument.” Electron. J. Differ. Equ., vol. 2017, p. 12, 2017.

[3] A. Bayramov, Z. Oer, and O. Baykal, "On identity for eigenvalues of second order differential operator equation." Math. Comput. Modelling, vol. 49, no. 3-4, pp. 403-412, 2009.

[4] L. A. Dikii, "A new method for calculation of eigenvalues of sturm-liouville problem," Doklady Akademii nauk SSSR, vol. 156, pp. 12-14, 1957.

[5] L. A. Dikii, "Trace formulas for sturm-liouville differential operators," Uspekhi Matematicheskikh Nauk, vol. 13, no. 3, pp. 111-143, 1958.

[6] I. Gel'fand and B. Levitan, "On a simple identity for the characteristic values of a differential operator of the second order differential equations," Dokl Akad. Nauk SSSR, vol. 88, pp. 593-596, 1953.

[7] M. L. Gorbachuk,, Boundary value problems for operator differential equations. Springer Science \& Business Media, 2012, vol. 48.

[8] N. J. Guliyev, "The regularized trace formula for the Sturm-Liouville equation with spectral parameter in the boundary conditions." Proc. Inst. Math. Mech., Natl. Acad. Sci. Azerb., vol. 22, pp. 99-102, 2005.

[9] F. Hıra and N. Altınışı, "The regularized trace of Sturm-Liouville problem with discontinuities at two points." Inverse Probl. Sci. Eng., vol. 25, no. 6, pp. 785-794, 2017.

[10] P. D. Lax, "Trace formulas for the Schrödinger operator." Commun. Pure Appl. Math., vol. 47, no. 4, pp. 503-512, 1994.

[11] A. Makin, "Regularized trace of the Sturm-Liouville operator with irregular boundary conditions." Electron. J. Differ. Equ., vol. 2009, p. 8, 2009.

[12] F. Maksudov, M. Bayramoglu, and E. Adigüzelov, "On the regularized trace of the SturmLiouville operator with an unbounded operator coefficient on a finite interval." Sov. Math., Dokl., vol. 30, pp. 169-173, 1984.

[13] V. Marchenko, Sturm-Liouville operators and applications, Revised ed. of the 1986 ed. ed. Providence, RI: AMS Chelsea Publishing, 2011.

[14] V. A. Sadovnichiǐ and V. E. Podol'skiı̌, "Traces of operators." Russ. Math. Surv., vol. 61, no. 5, pp. 885-953, 2006.

[15] J.-J. Sansuc and V. Tkachenko, "Characterization of the periodic and anti-periodic spectra of nonselfadjoint Hill's operators." in New results in operator theory and its applications: the Israel M. Glazman memorial volume. Basel: Birkhäuser, 1997, pp. 216-224. 
Authors' addresses

\section{F. Aydin Akgun}

Yildiz Technical University, Department of Mathematical Engineering, Istanbul, Turkey

E-mail address: fakgun@yildiz.edu.tr

\section{Bayramoglu}

Institute of Academy of Sciences of Azerbaijan, Baku, Azerbaijan

E-mail address: mamed.bayramoglueyahoo.com

\section{A. Bayramov}

Institute of Academy of Sciences of Azerbaijan, Baku, Azerbaijan

E-mail address: azadbay@gmail.com 